\title{
A Unified Model for Fuzzy Aggregation Operators and its Application in Group Decision Making
}

\author{
José M. Merigó \\ Department of Business Administration, University of Barcelona \\ Av. Diagonal 690, 08034 Barcelona, Spain
}

\begin{abstract}
We develop a new model for fuzzy decision making based on the use of several sources of information in the analysis. We introduce the fuzzy unified aggregation operator (FUAO). It is an aggregation operator that unifies other aggregation operators in the same formulation and considering the degree of importance of each concept in the analysis. Moreover, it also deals with uncertain environments that can be assessed with fuzzy numbers. We study the applicability of this new approach and we see that it is very broad. We focus on a fuzzy multi-person decision making problem regarding the selection of strategies.
\end{abstract}

Keywords: Fuzzy numbers; OWA operator; Weighted average; Probability; Decision making

\section{Introduction}

The aggregation operators are very common in our lifes [1,4,20-21]. One of the most common ones is the weighted average and the probability. They aggregate the information giving different weights to the information. They have been used in an extremely wide range of disciplines [6-7,17,27]. Another very useful aggregation operator is the ordered weighted averaging (OWA) operator [22]. Its main advantage is that it provides a parameterized family of aggregation operators between the minimum and the maximum. Since its introduction it has been used in a wide range of applications $[11,15,18,29-30,33]$.

These aggregations can be used individually but they can also be formulated in a unified model that includes them as particular cases. Recently, Merigó [6] introduced the probabilistic OWA weighted average (POWAWA) operator. It unifies the probability, the weighted average and the OWA operator in the same formulation and considering the degree of importance that each concept has in the aggregation.

However, the POWAWA operator only considers one weighting vector for the probability, one for the weighted average and one for the OWA operator. This formulation is practical for simple problems where we do not have many sources of information. However, it seems to be incomplete when we deal with complex environments affected by a wide range of sources of information.

In order to overcome this issue, Merigó [8] has suggested the use of the unified aggregation operator
(UAO). It includes the POWAWA operator as a particular case and a lot of other aggregation operators. Its main advantage is that we can use a lot of sources of information including several probabilistic or weighted aggregations in the analysis.

One limitation of the previous models is that they assume that the available information is clearly known and can be assessed with exact numbers. However, in real world problems we may find a lot of situations where the available information is imprecise and can not be assessed with exact numbers. Therefore, it is necessary to use another approach such as the use of fuzzy numbers (FNs).

The objective of this paper is to introduce a new model that it is able to deal with fuzzy environments when dealing with the UAO operator. Therefore, we introduce the fuzzy UAO (FUAO) operator. Its main advantage is that it can assess very complex environments by using a wide range of aggregation operators and in an uncertain environment where the imprecise information can be represented by using FNs. Moreover, it can represent the degree of importance that each fuzzy aggregation operator has in the analysis. It includes a wide range of aggregation operators such as the fuzzy weighted average (FWA), the fuzzy OWA (FOWA) operator, the fuzzy probabilistic aggregation (FPA), the fuzzy average (FA), the fuzzy POWAWA (FPOWAWA) operator and many others.

We study the applicability of this approach and we see that it is extremely broad because all the previous studies that use the average, the weighted average, the probability, the OWA operator or a related aggregation operator can be revised and extended with this new approach because we can always reduce the model to the classical approach. We focus on a fuzzy multi-person decision making problem regarding the selection of strategies. By using a multi-person analysis we are able to formulate the multi-person FUAO (MP-FUAO) operator. Its main advantage is that it can assess the FUAO operator when there is an interaction between the opinion of several persons in the analysis. It includes a wide range of particular cases such as the multi-person FPOWAWA (MP-FPOWAWA) operator and the multi-person fuzzy probabilistic weighted averaging (MP-FPWA) operator.

This paper is organized as follows. In Section 2 we briefly describe the some basic concepts regarding the FNs, the weighted aggregation operators, the OWA operator and the UAO operator. In Section 3 we introduce the FUAO operator and in Section 4 we develop an application in a fuzzy multi-person decision making problem. In Section 5 we present an illustrative example and 
in Section 6 we summarize the main conclusions of the paper.

\section{Preliminaries}

In this Section we briefly review some basic concepts regarding the fuzzy numbers, the weighted aggregation operators, the OWA operator and the UAO operator.

\subsection{The fuzzy numbers}

A FN $A$ is defined as a fuzzy subset [31] of a universe of discourse that is both convex (i.e., $\mu_{A}\left(\lambda x_{1}+(1-\lambda) x_{2}\right.$ $\geq \min \left(\mu_{A}\left(x_{1}\right), \mu_{A}\left(x_{2}\right)\right)$; for $\forall x_{1}, x_{2} \in R$ and $\left.\lambda \in[0,1]\right)$ and normal (i.e., $\sup _{x \in R} \mu_{A}(x)=1$ ).

Note that the FN may be considered as a generalization of the interval number although it is not strictly the same because the interval numbers may have different meanings. In the literature, we find a wide range of FNs $[2-3,5-7,10,12,32]$ such as the triangular FNs (TFNs), the trapezoidal FNs (TpFNs), the interval-valued FNs (IVFNs), the intuitionistic FNs (IFNs) and the generalized FNs (GFNs).

For example, a TpFN $A$ of a universe of discourse $R$ can be characterized by a trapezoidal membership function ( $\alpha$-cut representation) $A=(\underline{a}, \bar{a})$ such that

$$
\begin{aligned}
& \underline{a}(\alpha)=a_{1}+\alpha\left(a_{2}-a_{1}\right), \\
& \bar{a}(\alpha)=a_{4}-\alpha\left(a_{4}-a_{3}\right) .
\end{aligned}
$$

where $\alpha \in[0,1]$ and parameterized by $\left(a_{1}, a_{2}, a_{3}, a_{4}\right)$ where $a_{1} \leq a_{2} \leq a_{3} \leq a_{4}$, are real values. Note that if $a_{1}=$ $a_{2}=a_{3}=a_{4}$, then, the FN is a crisp value and if $a_{2}=a_{3}$, the FN is represented by a TFN. Note that the TFN can be parameterized by $\left(a_{1}, a_{2}, a_{4}\right)$.

In the following, we are going to review some basic FN arithmetic operations as follows. Let $A$ and $B$ be two TFNs, where $A=\left(a_{1}, a_{2}, a_{3}\right)$ and $B=\left(b_{1}, b_{2}, b_{3}\right)$. Then:

1. $A+B=\left(a_{1}+b_{1}, a_{2}+b_{2}, a_{3}+b_{3}\right)$

2. $A-B=\left(a_{1}-b_{3}, a_{2}-b_{2}, a_{3}-b_{1}\right)$

3. $A \times k=\left(k \times a_{1}, k \times a_{2}, k \times a_{3}\right)$; for $k>0$.

Note that other operations could be studied but in this paper we will focus on these ones. For more complete overviews about FNs, see for example [3,5-6].

\subsection{Weighted aggregation operators}

Weighted aggregation operators are those functions that weight the aggregation process by using the weighted average. Some examples are the aggregation with the weighted average or with belief structures that use the weighted average $[9,16,23]$. The weighted average can be defined as follows.

Definition 1. A WA operator of dimension $n$ is a mapping WA: $R^{n} \rightarrow R$ that has an associated weighting vector $W$, with $w_{i} \in[0,1]$ and $\sum_{i=1}^{n} w_{i}=1$, such that

$$
W A\left(a_{1}, \ldots, a_{n}\right)=\sum_{i=1}^{n} w_{i} a_{i}
$$

where $a_{i}$ represents the $i$ th argument variable.

Other extensions of the weighted average are those that use it with the OWA operator such as the WOWA operator and the hybrid averaging (HA) operator $[19,21]$.

\subsection{The OWA operator}

The OWA operator was introduced by Yager [22] and it provides a parameterized family of aggregation operators between the maximum and the minimum. It can be defined as follows.

Definition 2. An OWA operator of dimension $n$ is a mapping $O W A: R^{n} \rightarrow R$ that has an associated weighting vector $W$ of dimension $n$ with $\sum_{j=1}^{n} w_{j}=1$ and $w_{j} \in$ $[0,1]$, such that:

$$
O W A\left(a_{1}, a_{2}, \ldots, a_{n}\right)=\sum_{j=1}^{n} w_{j} b_{j}
$$

where $b_{j}$ is the $j$ th largest of the $a_{i}$.

Note that it is possible to distinguish between the descending OWA (DOWA) operator and the ascending OWA (AOWA) operator [22]. The OWA operator is commutative, monotonic, bounded and idempotent. Different types of OWA operators can be used in the analysis by using a different expression of the weighting vector $[13,17,24]$.

\subsection{The unified aggregation operator}

The unified aggregation operator (UAO) [8] is an aggregation operator that unifies a wide range of other aggregation operators in the same formulation and considering the degree of importance that each concept has in the aggregation. Its main advantage is that it can represent the information in a more complete way because it can deal with a wide range of aggregations that consider several sources of information. It can be defined as follows.

Definition 3. A unified aggregation operator of dimension $m$ is a mapping UAO: $R^{m} \times R^{n} \rightarrow R$, that has an associated weighting vector $C$ of dimension $m$ representing concepts with a degree of importance of $C_{h}$, such that:

$$
U A O\left(a_{1}, \ldots, a_{n}\right)=\sum_{h=1}^{m} \sum_{i=1}^{n} C_{h} w_{i}^{h} a_{i}
$$

where $C_{h}$ is the degree of importance that each concept has in the aggregation with $C_{h} \in[0,1]$ and $\sum_{h=1}^{m} C_{h}=1, w_{i}^{h}$ is the $i$ th weight of the $h$ th weighting vector $W$ with $w_{i}^{h} \in[0,1]$ and $\sum_{i=1}^{n} w_{i}^{h}=1$. 
The UAO operator is monotonic, bounded and idempotent. Note that if some of the aggregation operators used are not ordered according to $i$, then, we have to adapt the reordering of this aggregation to the initial ordering.

\section{The fuzzy unified aggregation operator}

The fuzzy unified aggregation operator (FUAO) is an aggregation operator that unifies other fuzzy aggregation operators in the same formulation and considering the degree of importance that each concept has in the aggregation. Moreover, it is able to assess uncertain environments that can not be assessed with exact numbers but it is possible to use FNs. Thus, we can represent the imprecise information considering the minimum and he maximum and the possibility that the internal values will occur. Furthermore, it can represent the information in a more complete way because it can deal with a wide range of aggregations that consider subjective and objective information, the attitudinal character of the decision maker and a lot of other concepts. It can be defined as follows.

Definition 4. Let $\Psi$ be the set of FNs. A FUAO operator of dimension $m$ is a mapping FUAO: $\Psi^{m} \times \Psi^{n} \rightarrow \Psi$, that has an associated weighting vector $C$ of dimension $m$ representing concepts with a degree of importance of $C_{h}$, such that:

$$
\operatorname{FUAO}\left(\tilde{a}_{1}, \ldots, \tilde{a}_{n}\right)=\sum_{h=1 i=1}^{m} \sum_{h}^{n} \tilde{C}_{h} \tilde{w}_{i}^{h} \tilde{a}_{i}
$$

where $\tilde{a}_{i}$ are the arguments represented in the form of FNs, $\tilde{C}_{h}$ is the degree of importance that each concept has in the aggregation with $\tilde{C}_{h} \in[0,1]$ and $\sum_{h=1}^{m} \tilde{C}_{h}=1, \tilde{w}_{i}^{h}$ is the $i$ th weight of the $h$ th weighting vector $W$ with $\tilde{w}_{i}^{h} \in[0,1]$ and $\sum_{i=1}^{n} \tilde{w}_{i}^{h}=1$.

Note that sometimes, it is not clear how to reorder the arguments. Thus, it is necessary to establish a criterion for ranking FNs. For simplicity, we recommend the following method. Select the FN with the highest value in its highest membership level, usually, when $\alpha=1$. Note that if the membership level $\alpha=1$ is an interval, then, we calculate the average of the interval. If there is still a tie, then, we recommend the use of an average or a weighted average of the FN according to the interests of the decision maker. Note that in the literature we find a lot of methods for ranking FNs [3,5-6].

The FUAO operator can be used with different types of FNs such as triangular FNs (TFNs), trapezoidal FNs (TpFNs), interval-valued FNs and type 2 FNs.

Note that if some of the aggregation operators used are not ordered according to $i$, such as the fuzzy OWA (FOWA) operator, then, we have to adapt the reordering of this aggregation (FOWA) to the initial ordering. That is, with the FOWA, we reorder the weights $w_{j}$ according to the initial positions of the arguments $i$.
Another interesting issue to consider is that if some of the weighting vectors $W^{h}$ or $C$ do not sum up to one, then, we have to normalize the information. Note that this situation is very common when the weighting vectors are represented with imprecise information such as interval or fuzzy numbers. That is:

$$
\operatorname{FUAO}\left(\tilde{a}_{1}, \ldots, \tilde{a}_{n}\right)=\frac{1}{\sum_{h=1}^{m} \tilde{C}_{h}} \sum_{h=1}^{m} \sum_{i=1}^{n} \frac{\tilde{C}_{h} \tilde{w}_{i}^{h} \tilde{a}_{i}}{\sum_{i=1}^{n} \tilde{w}_{i}^{h}}
$$

The FUAO operator is monotonic, bounded and idempotent. It is idempotent because if $\tilde{a}_{i}=a$, for all $i$, then, FUAO $\left(\tilde{a}_{1}, \ldots, \tilde{a}_{n}\right)=a$. It is monotonic because if $\tilde{a}_{i} \geq e_{i}$, for all $i$, then, FUAO $\left(\tilde{a}_{1}, \ldots, \tilde{a}_{n}\right) \geq \operatorname{FUAO}\left(e_{1}\right.$, $\left.\ldots, e_{n}\right)$. It is bounded because the FUAO aggregation is delimitated by the minimum and the maximum. Note that the boundary property is not always accomplished because it depends on the particular types of aggregation operators used. Thus, if we use for example a heavy OWA (HOWA) operator, then, the boundary property is not accomplished.

Another interesting issue is to analyze a wide range of particular types of FUAO operators. In this paper, we give special attention to situations that use the fuzzy probabilistic aggregation (FPA), the fuzzy weighted average (FWA) or the FOWA operator but it is worth noting that we could consider a lot of other aggregation operators including the use of induced aggregation operators $[14,17,28]$, norms [26] or generalized aggregation operators $[13,17,25]$.

First, we consider some basic cases such as the fuzzy probabilistic OWA weighted average (FPOWAWA) operator, the fuzzy probabilistic OWA (FPOWA), the fuzzy OWA weighted average (FOWAWA), the fuzzy probabilistic weighted average (FPWA), the FOWA, the FWA and the FPA. The FPOWAWA operator is obtained if we use the WA, the probability and the OWA in the same formulation. The FPOWAWA operator is formulated as follows:

$$
\begin{aligned}
\text { FPOWAWA } & \left(\tilde{a}_{1}, \ldots, \tilde{a}_{n}\right)= \\
& =\tilde{C}_{1} \sum_{j=1}^{n} \tilde{w}_{j} b_{j}+\tilde{C}_{2} \sum_{i=1}^{n} \tilde{v}_{i} \tilde{a}_{i}+\tilde{C}_{3} \sum_{i=1}^{n} \tilde{p}_{i} \tilde{a}_{i}
\end{aligned}
$$

Note that we could also express it as:

$$
f\left(\tilde{a}_{1}, \ldots, \tilde{a}_{n}\right)=\tilde{C}_{1} F O W A+\tilde{C}_{2} F W A+\tilde{C}_{3} F P A
$$

From this formulation, we could automatically obtain the other basic cases as follows:

- If $C_{1}=1$, we get the OWA (OWA) operator.

- If $C_{2}=1$, we get the WA.

- If $C_{3}=1$, we get the probabilistic aggregation (PA).

- If $C_{1}=0$, we form the probabilistic weighted average (PWA).

- If $C_{2}=0$, we form the probabilistic OWA (POWA) operator.

- If $C_{3}=0$, we form the OWAWA operator. 
Note that it is also possible to consider partial cases because $C_{1}, C_{2}$ and $C_{3}$, are interval numbers. Thus, in one part of the interval we can consider the FPOWAWA or any other case, nut not in the other part of the interval. For example, if we consider $C_{2}=[0,0,1]$, for the lower part of the interval we use a FPOWA operator and for the upper part the FPOWAWA operator.

Further interesting aggregations could be formed if we consider several different aggregations of the same type. Note that when using only one aggregation, we are implicitly assuming that we have summarized the information of two, three or more aggregations in one. That is:

$$
W A=C_{1} \times W A_{1}+C_{2} \times W A_{2}
$$

such that:

$$
\left(v_{1}, \ldots, v_{m}\right)=C_{1} \times\left(w_{1}, \ldots, w_{m}\right)+C_{2} \times\left(x_{1}, \ldots, x_{m}\right)
$$

with

$$
v_{i}=C_{1} \times w_{i}+C_{2} \times x_{i}
$$

In general, following Definition 4, we have the following expression when converting all the weighting vectors into a representative one:

$$
F U A O_{w}=\sum_{h=1}^{m} \tilde{C}_{h} \tilde{w}_{i}^{h}
$$

Note that if one of the weighting vectors does not sum up to one, have to use:

$$
F U A O_{w}=\frac{1}{\tilde{C} \tilde{W}^{h}} \sum_{h=1}^{m} \tilde{C}_{h} \tilde{w}_{i}^{h}
$$

From this, we can develop a wide range of methods for transforming a simple weighting vector into a more complex structure. Among others, we could mention Dempster-Shafer belief structures, hierarchical aggregations and multi-person aggregations $[9,14]$. Note that with aggregations that develop a reordering process such as the OWA operator, we have to adapt the reordering to the initial one in order to aggregate the weights as explained in Eq. (13).

If we consider the use of two weighted averages, a typical situation when using two different subjective sources of information, we get the following:

$$
F U A O\left(\tilde{a}_{1}, \ldots, \tilde{a}_{n}\right)=\tilde{C}_{1} F W A 1+\tilde{C}_{2} F W A 2
$$

If we use three weighted averages, we get:

$$
F U A O\left(\tilde{a}_{1}, \ldots, \tilde{a}_{n}\right)=\tilde{C}_{1} F W A 1+\tilde{C}_{2} F W A 2+\tilde{C}_{3} F W A 3(15)
$$

And so on. Note that we put a number to each FWA in order to distinguish each fuzzy weighted average.
The same analysis could be developed for the fuzzy probabilistic aggregation (FPA). For example, for three FPAs, we obtain:

$F U A O\left(\tilde{a}_{1}, \ldots, \tilde{a}_{n}\right)=C_{1} F P A 1+C_{2} F P A 2+C_{3} F P A 3$

We can also develop a similar analysis for the FOWA operator by forming two, three and more FOWAs in the aggregation. For example, for four FOWA aggregations we get the following aggregation operator:

$F U A O\left(\tilde{a}_{1}, \ldots, \tilde{a}_{n}\right)=$
$=\tilde{C}_{1} F O W A 1+\tilde{C}_{2} F O W A 2+\tilde{C}_{3} F O W A 3+\tilde{C}_{4} F O W A 4$

A similar methodology can be developed with the induced OWA (IOWA) operator [12]. For example, the FIOWA2 is formulated as follows:

FUAO $\left(\tilde{a}_{1}, \ldots, \tilde{a}_{n}\right)=\tilde{C}_{1}$ FIOWA $1+\tilde{C}_{2}$ FIOWA 2

Next, we present some other aggregations that use FOWAs, FWAs and FPAs in the same formulation. For example, let us look to the FP2OWAWA2 operator. That is, an aggregation operator that uses two probabilistic vectors, one WA and one OWA:

$$
\begin{aligned}
& F U A O\left(\tilde{a}_{1}, \ldots, \tilde{a}_{n}\right)= \\
& =\tilde{C}_{1} F P A 1+\tilde{C}_{2} F P A 2+\tilde{C}_{3} F O W A 1+\tilde{C}_{4} F W A 1+\tilde{C}_{5} F W A 2
\end{aligned}
$$

Another interesting case is the FPOWAWA2 operator that uses one FPA, one FOWA and two FWAs:

$$
\begin{aligned}
& F U A O\left(\tilde{a}_{1}, \ldots, \tilde{a}_{n}\right)= \\
& =\tilde{C}_{1} F P A 1+\tilde{C}_{2} F O W A 1+\tilde{C}_{3} F W A 1+\tilde{C}_{4} F W A 2
\end{aligned}
$$

In this way, we could consider more and more complex fuzzy aggregations such as the FP2OWA2WA2, the FP3OWA3WA3, and so on. Finally, we could imagine extremely complex situations where we consider hundreds, thousands, etc., of fuzzy aggregations in the analysis like a FP400OWA200WA700 operator. Note that these fuzzy aggregations are very common in the real world because the real high quality analysis that really represents the world should be assessed with this type of constructions. The situation in the present is that a lot of problems that should be assessed with these models are treated in an intuitionistic way. That is, people make analysis and decisions with their intuition and with some partial information that they have. Thus, we make some kind of simplification similar to the transformation explained in Eq. (12) and (13). But the real model behind this should be assessed with the FUAO operator.

Some simpler constructions could be considered by only using two of these concepts, thus obtaining the FPWA, the FPOWA or the FOWAWA operator. For example, we could form the FP2WA2 aggregation: 


$$
\begin{aligned}
& F U A O\left(\tilde{a}_{1}, \ldots, \tilde{a}_{n}\right)= \\
& \quad=\tilde{C}_{1} F P A 1+\tilde{C}_{2} F P A 2+\tilde{C}_{3} F W A 1+\tilde{C}_{4} F W A 2
\end{aligned}
$$

Or the FP2OWA2 aggregation:

$$
\begin{aligned}
& F U A O\left(\tilde{a}_{1}, \ldots, \tilde{a}_{n}\right)= \\
& =\tilde{C}_{1} F P A 1+\tilde{C}_{2} F P A 2+\tilde{C}_{3} F O W A 1+\tilde{C}_{4} F O W A 2
\end{aligned}
$$

Or the FOWAWA3 operator:

$$
\begin{aligned}
& F U A O\left(\tilde{a}_{1}, \ldots, \tilde{a}_{n}\right)= \\
& =\tilde{C}_{1} F O W A 1+\tilde{C}_{2} F W A 1+\tilde{C}_{3} F W A 2+\tilde{C}_{4} F W A 3
\end{aligned}
$$

In a similar way, we could construct a lot of other aggregation operators depending on the specific necessities needed in the analysis.

Some other interesting formulations are found when the FPA, the FWA or the FOWA become the fuzzy arithmetic mean (FAM). That is, when $w_{i}=1 / n$ for all $i$. If the FPA becomes the FAM, then we get the FAMOWAWA operator. If the FWA becomes the FAM, we form the FPOWAAM operator. And if the FOWA becomes the FAM, we get the FPAMWA operator:

Some other cases are found if some of the weighting vectors become the AM but some others do not. For example, in the FP2OWAWA3 we could assume that one FPA, and two FWA become the FAM thus obtaining the FPOWAWAAM3 operator:

$$
\begin{aligned}
& F U A O\left(\tilde{a}_{1}, \ldots, \tilde{a}_{n}\right)= \\
& =\begin{array}{l}
\tilde{C}_{1} F P A+\tilde{C}_{2} F O W A+\tilde{C}_{3} F W A 1+ \\
\tilde{C}_{4} F A M 1+\tilde{C}_{5} F A M 2+\tilde{C}_{6} F A M 3
\end{array}
\end{aligned}
$$

Note that the FAM provides the same result in all the cases, thus, we could simplify this formulation as follows:

$$
\begin{aligned}
& F U A O\left(\tilde{a}_{1}, \ldots, \tilde{a}_{n}\right)= \\
& \quad \tilde{C}_{1} F P A+\tilde{C}_{2} F O W A+\tilde{C}_{3} F W A 1+\left(\tilde{C}_{4}+\tilde{C}_{5}+\tilde{C}_{6}\right) F A M(25)
\end{aligned}
$$

Other interesting situations appear when we use the fuzzy maximum and the fuzzy minimum in the FOWA aggregation because we are establishing the bounds accepting the information obtained in the FWA and in the FPA. For example, in the FPOWA3WA2 operator we could use the maximum, the minimum and the FPOWAWA2 operator, thus obtaining:

$$
F U A O\left(\tilde{a}_{1}, \ldots, \tilde{a}_{n}\right)=\begin{aligned}
& \tilde{C}_{1} F P A+\tilde{C}_{2} F O W A+\tilde{C}_{3} F W A 1+ \\
& \tilde{C}_{4} F W A 2+\tilde{C}_{5} F M a x+\tilde{C}_{6} F M i n
\end{aligned}
$$

Note that a lot of other semi-boundary conditions appear depending on the type of aggregation operator used in the problem. Note also that by using several families of FOWA operators, we could form a lot of other cases such as the step-FOWA, the olympicFOWA and the centered-FOWA $[17,24]$.

\section{Fuzzy multi-person decision making with the FUAO operator}

The FUAO operator can be implemented in a lot of fields including statistics, decision-making, engineering, biology, physics and economics. In summary, all of the studies that use the probability, the weighted average or the OWA, can be revised and extended with this new approach.

In this paper, we focus on a fuzzy multi-person decision-making application in strategic management regarding the selection of general strategies in a company. Note that the use of a fuzzy multi-person analysis provides a deeper knowledge of the problem because usually, the decisions involve the opinion of a lot of people. The process to follow in the selection of general strategies with the FUAO operator in fuzzy multiperson decision making can be summarized as follows.

Step 1: Let $A=\left\{A_{1}, A_{2}, \ldots, A_{r}\right\}$ be a set of finite alternatives, and $C=\left\{S_{1}, S_{2}, \ldots, S_{n}\right\}$, a set of finite states of nature, forming the matrix $\left(\tilde{a}_{g i}\right)_{m \times n}$. Let $E=\left\{E_{1}, E_{2}\right.$, $\left.\ldots, E_{q}\right\}$ be a finite set of decision makers. Let $Z=\left(z_{1}\right.$, $\left.z_{2}, \ldots, z_{q}\right)$ be the weighting vector of the decision makers such that $\sum_{k=1}^{q} z_{k}=1$ and $z_{k} \in[0,1]$. Each decision maker provides their own payoff matrix $\left(\tilde{a}_{g i}{ }^{(k)}\right)_{r \times n}$.

Step 2: Calculate the weighting vectors of the FPA, FWA and FOWA to be used in the FUAO aggregation. In general, we assume the following $h$ th weighting vector for the FUAO: $W^{h}=\left(w_{1}^{h}, w_{2}^{h}, \ldots, w_{n}^{h}\right)$ such that $\sum_{i=1}^{n} w_{i}^{h}=1$ and $w_{i}^{h} \in[0,1]$.

Step 3: Use another FUAO (for simplicity we consider a FWA) to aggregate the information of the decision makers $E$ by using the weighting vector $Z=\left(z_{1}, z_{2}\right.$, $\left.\ldots, z_{q}\right)$. The result is the collective payoff matrix $\left(\tilde{a}_{g i}\right)_{r \times n}$. Thus, $x_{g i}=\sum_{k=1}^{q} z_{k} \tilde{a}_{g i}^{k}$.

Step 4: Calculate the aggregated results by using the FUAO operator explained in Eq. (5). Consider different particular cases of the FUAO operator in order to obtain a better representation of the decision process.

Step 5: Adopt decisions according to the results found in the previous steps. Select the alternative/s that provides the best result/s and establish a ranking of the alternatives.

Note that this aggregation process can be summarized using the following fuzzy aggregation operator that we call the multi-person - FUAO (MP-FUAO) operator.

Definition 5. Let $\Psi$ be the set of FNs. A MP-FUAO operator is a mapping MP-UAO: $\Psi^{n} \times \Psi^{n} \rightarrow \Psi$ that has a weighting vector $Z$ of dimension $q$ with $\sum_{k=1}^{q} z_{k}=1$ and $z_{k} \in[0,1]$ and $m$ weighting vectors $W$ of dimension $n$ with $\sum_{i=1}^{n} w_{i}^{h}=1$ and $w_{i}^{h} \in[0,1]$, such that: 


$$
\begin{aligned}
& \operatorname{MP-FUAO}\left(\left(\tilde{a}_{1}{ }^{1}, \ldots, \tilde{a}_{1}{ }^{q}\right), \ldots,\left(\tilde{a}_{n}{ }^{1}, \ldots, \tilde{a}_{n}{ }^{q}\right)\right)= \\
& =\sum_{h=1}^{m} \sum_{i=1}^{n} \tilde{C}_{h} \tilde{w}_{i}^{h} \tilde{a}_{i}
\end{aligned}
$$

where $\tilde{C}_{h}$ is the degree of importance that each concept has in the aggregation with $\tilde{C}_{h} \in[0,1]$ and $\sum_{h=1}^{m} \tilde{C}_{h}=1, \tilde{a}_{i}=\sum_{k=1}^{q} \tilde{z}_{k} \tilde{a}_{i}^{k}$ and $\tilde{a}_{i}^{k}$ is the argument variable provided by each person (or expert).

The MP-FUAO operator accomplishes similar properties than those explained in Section 3. Thus, we can find as special cases:

- The multi-person - FPOWAWA (MP-FPOWAWA) operator.

- The multi-person - FPA (MP-FPA) operator.

- The multi-person - FWA (MP-FWA) operator.

- The multi-person - FOWA (MP-FOWA) operator.

- The multi-person - FOWAWA (MP-FOWAWA).

- The multi-person - FP3OWA2WA3 operator.

- The multi-person - FP2OWA2WA3 operator.

- The multi-person - FP3000WA200WA600.

- The multi-person - FP2000OWA4000WA9000.

- Etc.

The MP-FUAO operator accomplishes similar properties than the FUAO and related aggregation operators such as the FPOWAWA operator. Thus, in general, it is monotonic, idempotent and bounded. Note that these properties are not always fulfilled because we may find particular aggregation operators of the FUAO that does not accomplish them such as a heavy aggregation.

\section{Illustrative example}

In the following, we present an illustrative example of the new approach in a fuzzy multi-person decisionmaking problem regarding the selection of strategies.

Assume a company that operates in Europe and North America is planning its general strategy for the next year and they consider four possible alternatives:

- $A_{1}$ : Expand to the Asian market.

- $A_{2}$ : Expand to the South American market.

- $A_{3}$ : Expand to the African market.

- $A_{4}$ : Do not make any expansion.

After careful analysis of the information, the group of experts of the company establishes the following general information regarding the general strategies. They assume that the key factor that determines the benefits of the strategies depend on the state of nature that occurs in the future. In this example, we assume five potential states of nature that may occur $S=\left\{S_{1}, S_{2}\right.$, $\left.S_{3}, S_{4}, S_{5}\right\}$ :

- $S_{1}$ : Negative growth rate.

- $S_{2}$ : Growth rate close to 0 .

- $S_{3}$ : Positive growth rate.

- $S_{4}$ : High growth rate.

- $S_{5}$ : Very high growth rate.

The group of experts of the country is constituted by 3 persons, each offering their own opinion regarding the results obtained with each strategy considered. The results are shown in Tables 1, 2 and 3.

Next, we aggregate the information of the three experts to obtain a collective matrix. We use the FWA to obtain this matrix by assuming that $Z=(0.2,0.3,0.5)$. The results are shown in Table 4 .

Table 1: Expert 1.

\begin{tabular}{cccccc}
\hline & $S_{1}$ & $S_{2}$ & $S_{3}$ & $S_{4}$ & $S_{5}$ \\
\hline$A_{1}$ & {$[40,50,60]$} & {$[60,70,80]$} & {$[30,40,50]$} & {$[50,60,70]$} & {$[40,50,60]$} \\
$A_{2}$ & {$[10,20,30]$} & {$[50,60,70]$} & {$[70,80,90]$} & {$[60,70,80]$} & {$[20,30,40]$} \\
$A_{3}$ & {$[30,40,50]$} & {$[40,50,60]$} & {$[70,80,90]$} & {$[20,30,40]$} & {$[50,60,70]$} \\
$A_{4}$ & {$[20,30,40]$} & {$[30,40,50]$} & {$[50,60,70]$} & {$[60,70,80]$} & {$[70,80,90]$} \\
\hline
\end{tabular}

Table 2: Expert 2.

\begin{tabular}{cccccc}
\hline & $S_{1}$ & $S_{2}$ & $S_{3}$ & $S_{4}$ & $S_{5}$ \\
\hline$A_{1}$ & {$[10,20,30]$} & {$[60,70,80]$} & {$[30,40,50]$} & {$[30,40,50]$} & {$[70,80,90]$} \\
$A_{2}$ & {$[20,30,40]$} & {$[50,60,70]$} & {$[30,40,50]$} & {$[60,70,80]$} & {$[60,70,80]$} \\
$A_{3}$ & {$[40,50,60]$} & {$[40,50,60]$} & {$[70,80,90]$} & {$[30,40,50]$} & {$[20,30,40]$} \\
$A_{4}$ & {$[20,30,40]$} & {$[20,30,40]$} & {$[60,70,80]$} & {$[60,70,80]$} & {$[70,80,90]$} \\
\hline
\end{tabular}

Table 3: Expert 3.

\begin{tabular}{cccccc}
\hline & $S_{1}$ & $S_{2}$ & $S_{3}$ & $S_{4}$ & $S_{5}$ \\
\hline$A_{1}$ & {$[10,20,30]$} & {$[20,30,40]$} & {$[30,40,50]$} & {$[60,70,80]$} & {$[70,80,90]$} \\
$A_{2}$ & {$[30,40,50]$} & {$[50,60,70]$} & {$[30,40,50]$} & {$[40,50,60]$} & {$[60,70,80]$} \\
$A_{3}$ & {$[40,50,60]$} & {$[20,30,40]$} & {$[70,80,90]$} & {$[60,70,80]$} & {$[20,30,40]$} \\
$A_{4}$ & {$[20,30,40]$} & {$[30,40,50]$} & {$[40,50,60]$} & {$[60,70,80]$} & {$[60,70,80]$} \\
\hline
\end{tabular}


Table 4: Collective results.

\begin{tabular}{cccccc}
\hline & $S_{1}$ & $S_{2}$ & $S_{3}$ & $S_{4}$ & $S_{5}$ \\
\hline$A_{1}$ & {$[16,26,36]$} & {$[40,50,60]$} & {$[30,40,50]$} & {$[49,59,69]$} & {$[64,74,84]$} \\
$A_{2}$ & {$[23,33,43]$} & {$[50,60,70]$} & {$[38,48,58]$} & {$[50,60,70]$} & {$[52,62,72]$} \\
$A_{3}$ & {$[38,48,58]$} & {$[30,40,50]$} & {$[70,80,90]$} & {$[43,53,63]$} & {$[26,36,46]$} \\
$A_{4}$ & {$[20,30,40]$} & {$[27,37,47]$} & {$[48,58,68]$} & {$[60,70,80]$} & {$[65,75,85]$} \\
\hline
\end{tabular}

Table 6: Aggregated results

\begin{tabular}{lcccc}
\hline \multicolumn{1}{c}{ FMin } & FMax & FOWA & FUAO \\
\hline$A_{1}$ & {$[16,26,36]$} & {$[64,74,84]$} & {$[35,45,55]$} & {$[34.39,44.39,54.39]$} \\
$A_{2}$ & {$[23,33,43]$} & {$[52,62,72]$} & {$[39.7,49.7,59.7]$} & {$[39.49,49.49,59.49]$} \\
$A_{3}$ & {$[26,36,46]$} & {$[70,80,90]$} & {$[37,47,57]$} & {$[42.91,52.91,62.91]$} \\
$A_{4}$ & {$[20,30,40]$} & {$[65,75,85]$} & {$[39.5,49.5,59.5]$} & {$[39.24,49.24,59.24]$} \\
\hline
\end{tabular}

Now, we analyze how to establish the weighting vector. In this example, we assume that we have two different sources of fuzzy probabilistic information, three different sources of FWA and one FOWA operator. Note that for simplicity we assume that the weights are exact numbers. This information is shown in Table 5.

Table 5: Weighting vectors of the FUAO.

\begin{tabular}{cccccc}
\hline & $w_{1}$ & $w_{2}$ & $w_{3}$ & $w_{4}$ & $w_{5}$ \\
\hline FPA 1 & 0.3 & 0.2 & 0.2 & 0.2 & 0.1 \\
FPA2 & 0.2 & 0.2 & 0.4 & 0.1 & 0.1 \\
FOWA1 & 0.1 & 0.2 & 0.2 & 0.2 & 0.3 \\
FWA 1 & 0.2 & 0.2 & 0.2 & 0.3 & 0.1 \\
FWA2 & 0.4 & 0.2 & 0.2 & 0.1 & 0.1 \\
FWA3 & 0.3 & 0.3 & 0.2 & 0.1 & 0.1 \\
\hline
\end{tabular}

Next, we can aggregate the information using several particular cases of the FUAO operators. In this example, we consider the individual results obtained with each weighting vector, the fuzzy maximum, the fuzzy minimum, the FOWA and the FUAO aggregation. We assume the following degree of importance for each aggregation operator shown in Table 5: $C=(0.2,0.2,0.1$, $0.1,0.2,0.2)$. The results are shown in Table 6 .

As we can see, the optimal choice is $A_{3}$. However, sometimes it is interesting to establish a ranking of the strategies because the optimal choice is not always the same. The results are shown in Table 7.

Table 7: Ranking of the alternatives.

\begin{tabular}{|c|c|c|c|}
\hline & Ranking & & Ranking \\
\hline FMin & $\left.\left.\left.A_{3}\right\} A_{2}\right\} A_{4}\right\} A_{1}$ & FOWA & $\left.\left.\left.A_{2}\right\} A_{4}\right\} A_{3}\right\} A_{1}$ \\
\hline FMax & $\left.\left.\left.A_{3}\right\} A_{4}\right\} A_{1}\right\} A_{2}$ & FUAO & $\left.\left.{ }_{2}\right\} A_{4}\right\} A_{1}$ \\
\hline
\end{tabular}

Note that depending on the particular method used, the results may be different and leading to different decisions. However, with this new approach we can consider all the sources of information and the different scenarios that may occur and select the one in closest accordance with our interests.

\section{Conclusions}

We have introduced the FUAO operator. It is a new general framework for unifying a wide range of aggregation operators in an uncertain environment that can be assessed with FNs. Its main advantage is that it unifies several aggregation operators such as the FPA, the FWA and the FOWA in the same formulation and considering the degree of importance that each concept has in the aggregation. Moreover, it can represent the real world in a more complete way because it can consider several sources of information, a typical situation when analyzing the heterogeneity of the real world. We have seen that it includes a wide range of particular cases including the FPA, the FWA, the FOWA, the FPOWAWA, the FPWA, the FPOWA, the FOWAWA, the FP3OWA2WA4 and many others.

We have studied the applicability of the new approach and we have seen that it is very broad because all the previous studies that use the PA, the WA or the OWA can be revised and extended with this formulation. We have focused on a fuzzy multi-person decision making problem concerning strategic management. By using a multi-person analysis, we have formed the MPFUAO operator that provides a more flexible formulation because it can deal with the opinion of several experts in the analysis.

In future research we expect to develop further developments to this approach by adding more concepts in the analysis such as the use generalized aggregation operators, distance measures and other forms of imprecise information.

\section{References}

[1] G. Beliakov, A. Pradera and T. Calvo, Aggregation Functions: A Guide for Practitioners, Berlin: Springer-Verlag, 2007.

[2] S.S.L. Chang and L.A. Zadeh, On fuzzy mapping and control, IEEE Transactions on Systems, Man and Cybernetics, 2: 30-34, 1972.

[3] D. Dubois and H. Prade, Fuzzy Sets and Systems: Theory and Applications, New York: Academic Press, 1980.

[4] M. Grabisch, J.L. Marichal, R. Mesiar, E. Pap, Aggregation Functions, Cambridge: Cambridge University Press, 2009.

[5] A. Kaufmann and M.M. Gupta, Introduction to Fuzzy Arithmetic, Rheinhold: Publications Van Nostrand, 1985.

[6] J.M. Merigó, New extensions to the OWA operators and its application in decision making (In Spanish). 
PhD Thesis, Department of Business Administration, University of Barcelona, 2008.

[7] J.M. Merigó, Fuzzy decision making with immediate probabilities, Computers \& Industrial Engineering, 58: 651-657, 2010.

[8] J.M. Merigó, Decision making with probabilities, weighted averages and OWA operators, Proceedings of the IEEE-SSCI 2011, Paris, France, (to be published).

[9] J.M. Merigó and M. Casanovas, Induced aggregation operators in decision making with DempsterShafer belief structure, International Journal of Intelligent Systems, 24: 934-954, 2009.

[10] J.M. Merigó and M. Casanovas, Fuzzy generalized hybrid aggregation operators and its application in decision making, International Journal of Fuzzy Systems, 12: 15-24, 2010.

[11] J.M. Merigó and M. Casanovas, Induced and heavy aggregation operators with distance measures, Journal of Systems Engineering and Electronics, 21: 431-439, 2010.

[12] J.M. Merigó and M. Casanovas, The fuzzy generalized OWA operator and its application in strategic decision making, Cybernetics \& Systems, 41: 359370, 2010.

[13] J.M. Merigó and M. Casanovas, The uncertain induced quasi-arithmetic OWA operator, International Journal of Intelligent Systems, 26: 1-24, 2011.

[14] J.M. Merigó and M. Casanovas, Decision making with distance measures and induced aggregation operators, Computers \& Industrial Engineering, 60: 66-76, 2011.

[15] J.M. Merigó and M. Casanovas, Induced and uncertain heavy OWA operators, Computers \& Industrial Engineering 60: 106-116, 2011.

[16] J.M. Merigó, M. Casanovas and L. Martínez, Linguistic aggregation operators for linguistic decision making based on the Dempster-Shafer theory of evidence, International Journal of Uncertainty, Fuzziness and Knowledge-Based Systems, 18: 287304, 2010.

[17] J.M. Merigó and A.M. Gil-Lafuente, The induced generalized OWA operator, Information Sciences, 179: 729-741, 2009.

[18] J.M. Merigó and A.M. Gil-Lafuente, New decision making techniques and their application in the se- lection of financial products, Information Sciences, 180: 2085-2094, 2010.

[19] V. Torra, The weighted OWA operator, International Journal of Intelligent Systems, 12: 153-166, 1997.

[20] V. Torra and Y. Narukawa, Modelling Decisions: Information Fusion and Aggregation Operators, Berlin: Springer-Verlag, 2007.

[21] Z.S. Xu and Q.L. Da, An overview of operators for aggregating information, International Journal of Intelligent Systems, 18: 953-968, 2003.

[22] R.R. Yager, On ordered weighted averaging aggregation operators in multi-criteria decision making, IEEE Transactions on Systems, Man Cybernetics B, 18: 183-190, 1988.

[23] R.R. Yager, Decision making under DempsterShafer uncertainties, International Journal of General Systems, 20: 233-245, 1992.

[24] R.R. Yager, Families of OWA operators, Fuzzy Sets and Systems, 59: 125-148, 1993.

[25] R.R. Yager, Generalized OWA aggregation operators, Fuzzy Optimization and Decision Making, 3: 93-107, 2004.

[26] R.R. Yager, Norms induced from OWA operators, IEEE Transactions on Fuzzy Systems, 18: 57-66, 2010.

[27] R.R. Yager, K.J. Engemann and D.P. Filev, On the concept of immediate probabilities, International Journal of Intelligent Systems, 10: 373-397, 1995.

[28] R.R. Yager and J. Kacprzyk, The Ordered Weighted Averaging Operators: Theory and Applications. Norwell: Kluwer Academic Publishers, 1997.

[29] R.R. Yager, J. Kacprzyk and G. Beliakov, Recent Developments on the Ordered Weighted Averaging Operators: Theory and Practice, Springer-Verlag, Berlin, 2011.

[30] L.A. Zadeh, Fuzzy sets, Information and Control, 8: 338-353, 1965.

[31] L.A. Zadeh, The concept of a linguistic variable and its application to approximate reasoning. Part 1 , Information Sciences, 8: 199-249; Part 2, Information Sciences, 8: 301-357, Part 3, Information Sciences, 9: 43-80, 1975.

[32] L.G. Zhou and H.Y. Chen, Generalized ordered weighted logarithm aggregation operators and their applications to group decision making. International Journal of Intelligent Systems, 25: 683-707, 2010. 